\title{
PRODUCTION CONTROL OF A PULL SYSTEM WITH PRODUCTION AND DEMAND UNCERTAINTY
}

\author{
Barıș Tan \\ Graduate School of Business, Koç University \\ e-mail:btan@ku.edu.tr
}

December 2001

\begin{abstract}
We consider a continuous material flow manufacturing system with an unreliable production system and a variable demand source which switches randomly between zero and a maximum level. The failure and repair times of the production system and the switching times of the demand source are assumed to be exponentially distributed random variables. The optimal production flow control policy that minimizes the expected average inventory carrying and backlog costs is characterized as a double-hedging policy. The optimal hedging levels are determined analytically by minimizing the closed-form expression of the cost function. We investigate two approximate single hedging policies. It is empirically shown that an approximate policy that uses a single hedging level which is the sum of a production uncertainty term and a demand uncertainty term gives accurate results for the expected average cost.
\end{abstract}

\section{Introduction}

The purpose of this study is to analyze a manufacturing flow control system with an unreliable manufacturing facility and uncertain demand which switches randomly between zero and a maximum value with exponential switching times. The randomly switching demand source in this model differentiates this study from a chain of related articles in the manufacturing flow control literature including (Kimemia and Gershwin (1983), Bielecki and Kumar (1988), Akella and Kumar (1986), Sharifnia (1988), Liberopoulos and Caramanis (1994), and Hu, Vakili, and Yu (1994)).

The system with two machine states and two demand states is transformed into an equivalent system with four states. Following the results of $\mathrm{Hu}$, Vakili, and $\mathrm{Yu}$ (1994) and Liberopoulos and Caramanis (1994), we argue that the optimal policy is a hedging point policy. When the maximum production rate is less than or equal to the maximum demand rate, the policy is a single hedging policy where the only feasible hedging point corresponds to the only feasible state where the machine is up and the demand is zero. If the maximum production rate is greater than the maximum demand rate, there are two feasible hedging points corresponding to the two feasible states, where the machine is up and the demand rate is either zero or at its maximum value. This leads to a double hedging policy. The steady-state distribution of inventory/backlog and state of the system is determined and utilized to find the closed form expressions for the optimal hedging point(s). 
We introduce two single hedging policy approximations. In the first approximation, a single optimal hedging point is derived for the original system. In the second approximation, the hedging is given as the sum of a demand uncertainty term and a production uncertainty term. The demand uncertainty term is determined by considering a system with a reliable station whose production rate is set to the stand-alone average production rate of the station in the original system and variable demand. Similarly the production uncertainty term is determined by considering a system with an unreliable station and constant demand source whose rate is set to the average demand rate of the original system. An intuitive closed-form expression for the single hedging point is given. We compare the average cost of these approximations with the optimal double hedging policy.

\section{Model Description}

We consider a continuous material flow system with a single unreliable station that produces to meet a random demand. The state of the station and the demand source at time $t$ is $(\alpha(t), \beta(t))$ where $\alpha(t)$ is the state of the station which is either up(U) or down (D) and $\beta(t)$ is the state of the demand source which is either on $(\mathrm{U})$ or off $(\mathrm{D})$. Then there are four different states: UU, UD, DU, DD. At time $t$, the amount of finished foods inventory or backlog is $x(t)$. Then the state of the system at time $t$ is $(\alpha(t), \beta(t), x(t))$.

The processing rate of the station at time $t$ is denoted by $u(t)$. The maximum processing rate of the station is $\mu_{1}$, i.e., $0 \leq u(t) \leq \mu_{1}$. The machine is unreliable and the time to failure is an exponentially distributed random variable with rate $p(t)$ which is linearly dependent on the processing rate $p(t)=u(t) p_{1} / \mu_{1}$. In other words, we assume operations dependent failures. The repair time is also an exponential random variable with rate $r_{1}$.

The demand rate at time $t$ is denoted by $d(t)$. The demand variability is modeled by a valve that is switched on and off randomly. When the valve is on, there is a constant stream of demand with rate $\mu_{2}$ and when the valve is off, there is no demand. The time to switch on and the time to switch off are also assumed to be exponentially distributed random variables with rates $p_{2}$ and $r_{2}$.

We define $e_{i}=\frac{r_{i}}{p_{i}+r_{i}}, i=1,2$ as the average up time of the station and the demand source. Then the average production rate is $E_{1}=e_{1} \mu_{1}$ and the average demand rate is $E_{2}=e_{2} \mu_{2}$. 


\section{Production Control Problem}

In the production control problem, the decision variable is the instantaneous processing rate of the station $u(t)$. The processing rate of the station $u(t)$ is controlled in real time to match capacity and demand as closely as possible to minimize the expected average inventory carrying and backlogging costs. The cost of holding inventory is $c^{+}$and the backlogging cost is $c^{-}$. Let $x^{+}(t)=\max \{x(t), 0\}$ and $x^{-}(t)=\max \{-x(t), 0\}$, then the production control problem can be stated as

$$
\operatorname{Min} E\left[\int_{0}^{\infty}\left(c^{+} x^{+}(t)+c^{-} x^{-}(t)\right) d t\right]
$$

subject to

$$
\begin{aligned}
& \frac{d}{d t} x(t)=u(t)-d(t) \\
& u(t)= \begin{cases}0 \leq u(t) \leq \mu_{1} & \text { if } \alpha(t)=\mathrm{U} \\
0 & \text { if } \alpha(t)=\mathrm{D}\end{cases} \\
& d(t)= \begin{cases}0 & \text { if } \beta(t)=\mathrm{U} \\
\mu_{2} & \text { if } \beta(t)=\mathrm{D}\end{cases}
\end{aligned}
$$

Markov dynamics for $\alpha(t)$ with rates $p(t)$ (from $\mathrm{U}$ to $\mathrm{D})$ and $r_{1}$ (from $\mathrm{D}$ to $\mathrm{U}$ )

Markov dynamics for $\beta(t)$ with rates $p_{2}$ (from $\mathrm{U}$ to $\left.\mathrm{D}\right)$ and $r_{2}$ (from $\mathrm{D}$ to $\mathrm{U}$ )

$x(0)$ specified.

\section{Characterization of the Optimal Production Control Policy}

Let us consider a station with four modes of operation where the modes UU, UD, DU, and DD correspond to the state of the original system and assume that the demand is constant with rate $\mu_{2}$. Furthermore let the maximum production rates for each mode be $\mu_{1}, \mu_{1}+\mu_{2}, 0$, and $\mu_{2}$ respectively. Then the maximum effective rates of increase in the surplus will be $\mu_{1}-\mu_{2},\left(\mu_{1}+\mu_{2}\right)-\mu_{2}=\mu_{2}, 0-\mu_{2}=-\mu_{2}$, and $\mu_{2}-\mu_{2}=0$ which are the same as in the original model. Therefore, this equivalent system can produce the same sample path for the surplus variable $x(t)$ as the system considered here. In this equivalent system, if $\mu_{1}>\mu_{2}$ only the modes UU and UD are feasible and if $\mu_{1} \leq \mu_{2}$, only the mode UD is feasible.

Hu, Vakili and Yu (1994) consider a single machine system with two machine states (up and down). The machine must produce in order to meet a constant demand rate. They show that for an 
extended discounted production inventory/backlog cost criterion, the optimal control policy is a hedging point policy, if and only if the failure rate of the machine is a linear function of the production rate. Liberopoulos and Caramanis (1994) conclude that the optimal control policy is a hedging point policy, if the failure rate of the machine is a concave or a linear function of the production rate for an expected average production inventory/backlog cost criterion. Based on these results, we believe that such policies are also likely to be optimal for our system, and we will restrain ourselves to hedging point policies in this paper.

We state the following feedback policy for the processing rate of the machine $u^{*}(x(t),[\alpha(t)$, $\beta(t)])$ as the optimal policy for this failure prone manufacturing system with variable demand. There are two hedging levels of this policy, an upper hedging level, $Z_{u}$ and a lower hedging level, $Z_{d}$. If the machine is up and the demand is on, the station produces with the maximum rate until the hedging point $Z_{u}$ is reached. When the surplus level is equal to $Z_{u}$, it produces with the demand rate:

$$
u^{*}(x, \mathrm{UU})=\left\{\begin{array}{cl}
\min \left(\mu_{1}, \mu_{2}\right) & x=Z_{u} \\
\mu_{1} & x<Z_{u} . \\
0 & x>Z_{u}
\end{array}\right.
$$

The second rule is related to the state where the machine is up and the demand is off. In this state, the station produces with the maximum processing rate until the hedging level $Z_{d}$ is reached. If this level is exceeded, the station halts production:

$$
u^{*}(x, \mathrm{UD})=\left\{\begin{array}{cc}
\mu_{1} & x<Z_{d} \\
0 & x \geq Z_{d}
\end{array} .\right.
$$

We assume that $Z_{d} \leq Z_{u}$. Note that this is a plausible assumption since the state UU is closer to an infeasible state than state UD. Therefore there is a greater need to hedge against uncertainty when the state is in UU and it has a higher hedging point. Since linear holding and backlog costs are assumed, both of these hedging levels are nonnegative (Liberopoulos and $\mathrm{Hu}, 1995$ ).

In order to analyze the performance of this production system governed by this optimal control policy, we first set the hedging levels to $Z_{u}$ and $Z_{d}$. Then we derive the cost function as a function of these parameters. Finally, the cost function is optimized for the optimal values of $Z_{u}$ and $Z_{d}$. This approach is similar to the one utilized in (Algoet, 1989, Sharifnia (1988) and Liberopoulos and 
Caramanis, 1994). The details of the derivation of the steady-state distribution and the optimal hedging levels are not reported here and can be found in (Tan, 2000).

\section{Approximation of the Optimal Policy}

The double-hedging policy is a dynamic policy that requires adjusting the production rate depending on the state of the demand. This introduces a difficulty in implementation due to observability of the demand state and also due to the need to adjust the production rate too frequently for rapidly changing demand. We consider two approximations of the optimal policy.

\subsection{Single Hedging Policy}

The first approximation is to use a single hedging level for the original system. Note that when $\mu_{1} \leq \mu_{2}$, the double-hedging policy reduces to a single hedging policy. Therefore this policy is an approximation only for the case $\mu_{1}>\mu_{2}$. The system controlled by this policy is again modeled as a state space model and the steady-state distribution is obtained. Then the cost function is optimized to determine the optimal single hedging level. In this case, although an analytic expression is available for the optimal single hedging level, it is too lengthy to get any insight from.

\subsection{Superposition Policy}

One plausible approximation is to assume that the optimal hedging level for the single hedging policy is the sum of the hedging levels for an uncertain production-deterministic demand case and a deterministic production-uncertain demand case. That is, a hedging level $Z_{P D}$ with value

$$
Z_{P D}^{*}=Z_{P}^{*}+Z_{D}^{*}
$$

where $Z_{P}^{*}$ is the uncertain production term and $Z_{D}^{*}$ is the uncertain demand term is used as an approximate hedging level.

In this section, the expression for the single hedging level is written in a way to show clearly the effects of system parameters including the variability of production and demand, average production and demand rate, and maximum production and demand rate. The variability of production and demand is summarized by using the steady-state squared coefficient of variation rate of the total production $N_{1}(t)$ and demand $N_{2}(t)$ accumulated during a period $[0, t]$. The asymptotic variance rate of the amount of material produced $V_{1}(t)$ and the asymptotic variance rate of the demand $V_{2}(t)$ can be written by using the result given in (Tan, 1997) as 


$$
V_{i}=\lim _{t \rightarrow \infty} \frac{\operatorname{Var}\left[N_{i}(t)\right]}{t}=\frac{2 p_{i} r_{i}}{\left(p_{i}+r_{i}\right)^{3}} \mu_{i}^{2}=\frac{2}{r_{i}}\left(1-e_{i}\right) e_{i}^{2} \mu_{i}^{2} \quad i=1,2 .
$$

then the squared coefficient of variation of production and demand are defined as:

$$
c v_{i}^{2}=\lim _{t \rightarrow \infty} \frac{V_{i}}{E_{i}^{2}} t=\frac{2}{r_{i}}\left(1-e_{i}\right) \quad i=1,2 .
$$

The above result is invoked to write the optimal hedging point in terms of the production and demand uncertainty.

\section{Uncertain Production Term}

In order to determine the uncertain production term, the demand is approximated with a constant flow whose rate is equal to the average demand rate $E_{2}$ and no information about its variability is used. This case is analyzed in detail in the literature (see, Gershwin, 1994). The optimal policy is a single hedging policy with hedging level $Z_{P}$. After adjusting for the production-rate dependent failure rate and setting the demand rate to $E_{2}=\mu_{2} \frac{r_{2}}{p_{2}+r_{2}}$ and rewriting the result in terms of the production variability yields the following uncertain production term

$$
Z_{P}^{*}=\max \left\{0,-c v_{1}^{2} \frac{e_{1}}{1-e_{1}} \frac{\left(\mu_{1}-E_{2}\right) E_{2}}{E_{1}-E_{2}} \ln \left[\left(\frac{c^{+}}{c^{+}+c^{-}}\right) \cdot \frac{e_{1}}{1-e_{1}} \cdot \frac{\mu_{1}-E_{2}}{E_{2}}\right]\right\}
$$

where $c v_{1}^{2}$ is given in equation (9).

The above expression shows that, keeping all the other system parameters the same, as the variability of the production $c v_{1}^{2}$ increases, the hedging level increases linearly. Similarly, the difference between the maximum production rate and the average demand rate, the difference between the average production and the average demand rate, and the average demand rate affect the hedging level. The optimal hedging level converges to $Z_{P}^{*}$ as $c v_{1}^{2}$ approaches 0 .

\section{Uncertain Demand Term}

In order to determine the uncertain demand term, we consider a case where the machine is perfectly reliable but the demand is uncertain. In this case, the production source is approximated with a flow whose maximum rate is the average production rate of the original station $E_{1}$, and no information about its variability is used. If this system is transformed to an equivalent system with a machine with two failure states and constant demand, it can be shown that the optimal policy is again 
a single hedging policy with hedging level $Z_{D}$. If $\mu_{1} e_{1}>\mu_{2}$, this approximation implies that there always exists enough capacity to meet the demand, and therefore the optimal hedging level is zero. Otherwise, the optimal hedging level can be determined from the steady-state distribution of the surplus variable for this system that yields

$$
Z_{D}^{*}=\left\{\max \left\{0,-c v_{2}^{2} \frac{e_{2}}{1-e_{2}} \frac{\left(\mu_{2}-E_{1}\right) E_{1}}{E_{1}-E_{2}} \ln \left[\left(\frac{c^{+}}{c^{+}+c^{-}}\right) \cdot \frac{E_{1}}{E_{2}}\right]\right\} \begin{array}{l}
E_{1} \leq \mu_{2} \\
0 \\
E_{1}>\mu_{2}
\end{array}\right.
$$

where $c v_{2}^{2}$ is given in equation (9).

The above expression shows that, keeping all the other system parameters the same, as the variability of the demand $c v_{2}^{2}$ increases, the hedging level increases linearly. Similarly, the difference between the maximum demand rate and the average production rate, the difference between the average production and the average demand, and the average production rate affect the hedging level. The optimal hedging level converges to $Z_{D}^{*}$ as $c v_{2}^{2}$ approaches $0\left(r_{2}\right.$ approaches infinity $)$.

\section{Numerical Results and Observations}

In this section we investigate the performance of the single hedging policy and the superposition approximation. In Figure 1, the optimal hedging levels for the optimal policy, and also the hedging levels for two approximate policies are given for different levels of production and demand variability for the case $\mu_{1}>\mu_{2}$. As the production and demand variability increase, the optimal hedging levels for the optimal policy and the approximate policies also increase to hold enough safety stock against uncertain production and demand. However, since the superposition approximation does not consider the interaction between the production and demand uncertainty, it sets a lower hedging point compared to the single hedging approximation. The optimal hedging level for the single hedging policy turns out to be very close to the upper hedging level $Z_{u}$. Since the backlog cost is usually higher than the inventory carrying cost, the first priority of the policy is to attain a reasonable safety stock. When the policy is restricted to have a single hedging level, this level turns out to be close to the maximum hedging level in the optimal policy.

Figure 2 shows the average percentage savings that will be obtained by using the optimal policy compared to the approximate single hedging policy for the case $\mu_{1}>\mu_{2}$. Figure 2 suggests that the approximate single hedging policy is quite accurate and yields very close results compared to the 
optimal policy. As Figure 1 shows, the single hedging level is close to the upper hedging level of the double hedging policy. This means that single hedging policy sets a comparable safety stock for possible future capacity shortages. The advantage of the double hedging policy comes from temporarily stopping the production in the region $Z_{d}<x<Z_{u}$ when the demand is down. However, the time spent in the state where the demand is down and $Z_{d}<x<Z_{u}$ is short compared to the time spent in the region $x<Z_{d}$. Consequently, the cost benefit that can be obtained in this short period has a smaller effect on the overall cost function. As a result, the single hedging policies perform quite well compared to the double hedging policy.

Figure 2 shows that the saving percentage increases as the demand variability increases. Following equation (9), the demand variability increases with the average switching time from zero to the maximum level for the same $e_{2}$. Therefore as the demand variability increases, the double hedging policy halts the production temporarily above $Z_{d}$ when the demand is down for a longer period of time until the demand switches to its maximum level. This action prevents unnecessary inventory buildup for a longer period. Thus the double hedging policy yields better performance compared to the single hedging approximation as the demand variability increases.

The advantage of the double hedging policy increases as the production uncertainty decreases for a given level of demand uncertainty. The explanation of this phenomenon follows a similar argument given above for the period of comparative advantage between the policies. As the production uncertainty increases, the expected repair time increases for the same $e_{1}$. Therefore the total up time of the machine when $Z_{d}<x<Z_{u}$ that will give the double hedging policy an advantage gets shorter and the advantage of the double hedging policy decreases as the production uncertainty increases.

Next we investigate the accuracy of the superposition policy where the hedging level is approximated by the sum of the hedging levels for uncertain production-deterministic demand and deterministic production-uncertain demand policies. Figure 3a, b, and c depict the average cost increase percentage when using the superposition policy for the cases $\mu_{1}>\mu_{2}, \mu_{1}=\mu_{2}$, and $\mu_{1}<\mu_{2}$. The figures suggest that the superposition approximation performs remarkably well. The maximum cost increases for the cases $\mu_{1}>\mu_{2}, \mu_{1}=\mu_{2}$, and $\mu_{1}<\mu_{2}$ are $1.65 \%, 0.13 \%$, and $0.035 \%$. For the case $\mu_{1}>\mu_{2}$, since the optimal policy is a double hedging policy, some of the cost increase is due to using a single hedging level instead of two levels. For the cases $\mu_{1} \leq \mu_{2}$, the superposition policy practically yields the 
optimal costs. Note that, the cost function is quite flat and insensitive to the changes in the hedging levels. This property of the cost function allows us to use this approximation and get very accurate results for the total cost.

\section{Conclusions}

In this study, a continuous material flow production system with production and demand uncertainty is analyzed. The production control policy is stated as a hedging-type policy where there are two hedging levels depending on the state of the demand. This policy performs better than the approximate policies that set a single hedging level. This preliminary analysis indicates that real-time policies that incorporate the state of the demand may yield slight improvements in the performance of a manufacturing system.

However, the difficulty of determining the state of the demand and also adjusting the production rate each time the state of the demand changes make it harder to implement the double-hedging policy. Therefore, two single hedging level policies are proposed. The first policy determines the optimal level of the single hedging level for the original model. In the second policy, the single hedging point is set to a level that is the sum of a production uncertainty term and a demand uncertainty term and therefore referred as the superposition approximation. It is shown empirically that the superposition approximation yields almost the same optimal cost for the case when $\mu_{1} \leq \mu_{2}$ and yields less than $1.7 \%$ cost increase when $\mu_{1}>\mu_{2}$. Although the optimal single hedging level in the first approximation can also be determined analytically, the expression for the superposition hedging level shows clearly the effects of system parameters including the variability of production and demand, average production and demand rate, and maximum production and demand rate. 

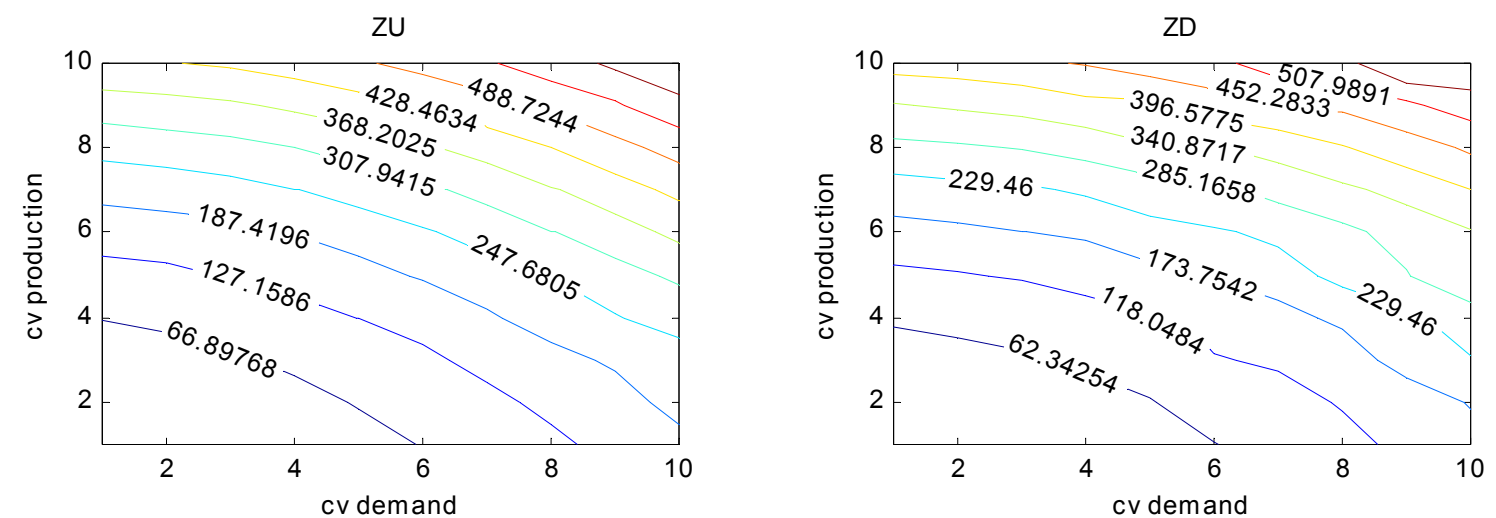

ZS
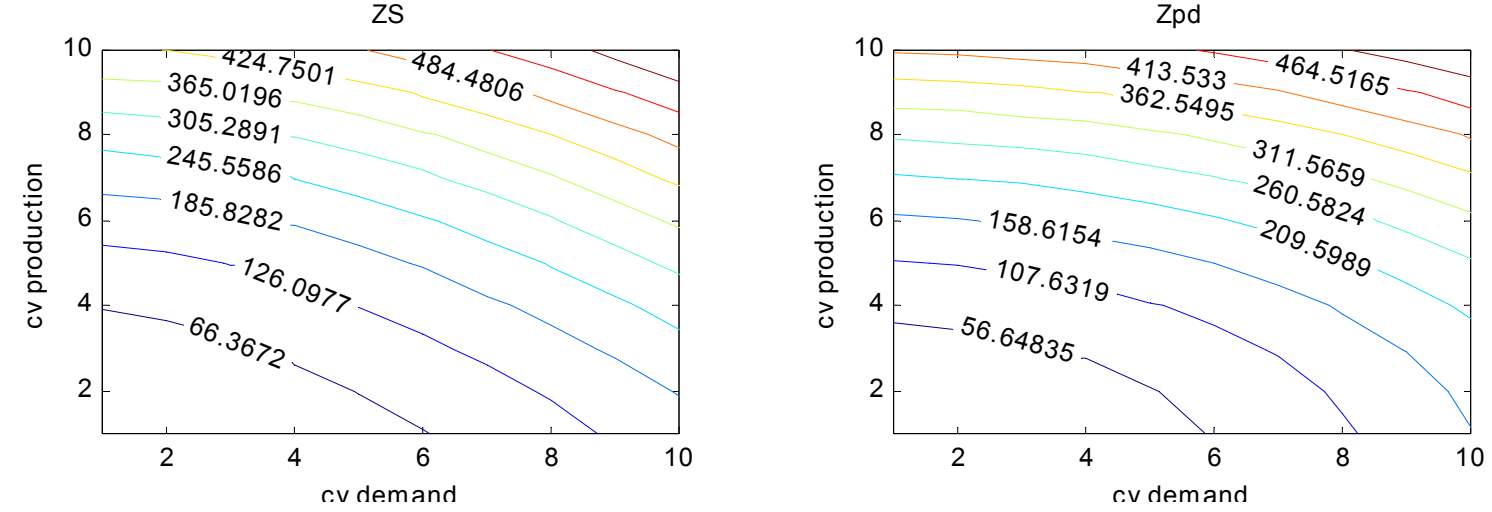

Figure 1. Hedging Levels for the optimal policy (ZU, ZD), single hedging policy (ZS), and the superposition policy $(\mathrm{Zpd})\left(\mu_{1}=1.0, E_{1}=0.9, \mu_{2}=0.95, E_{2}=0.8, c^{+}=1, c^{-}=2\right)$

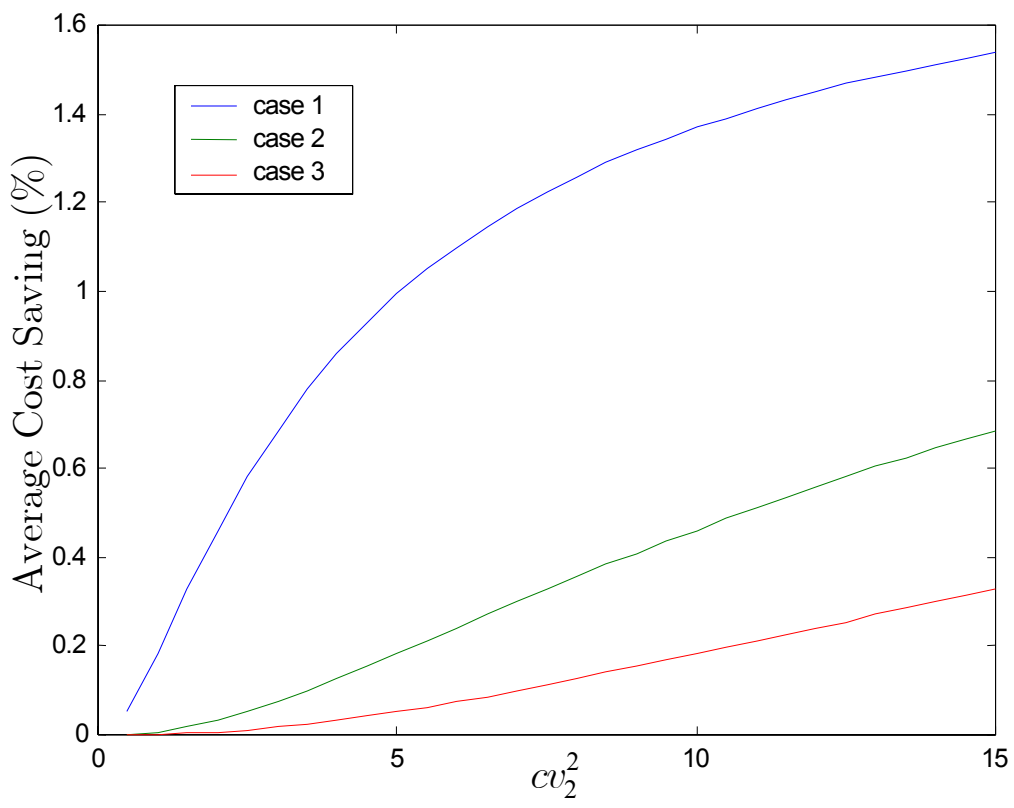

Figure 2. Average cost savings obtained by using double hedging policy instead of the single hedging policy vs. demand variability for the case $\mu_{1}>\mu_{2}\left(\left(\mu_{1}=2.0, E_{1}=1.4, \mu_{2}=1.0, E_{2}=0.8, c^{+}=1, \quad c^{-}\right.\right.$ $=2$, case $1: c v_{1}^{2}=1$, case $2: c v_{1}^{2}=5$, case $3: c v_{1}^{2}=10$ ). 


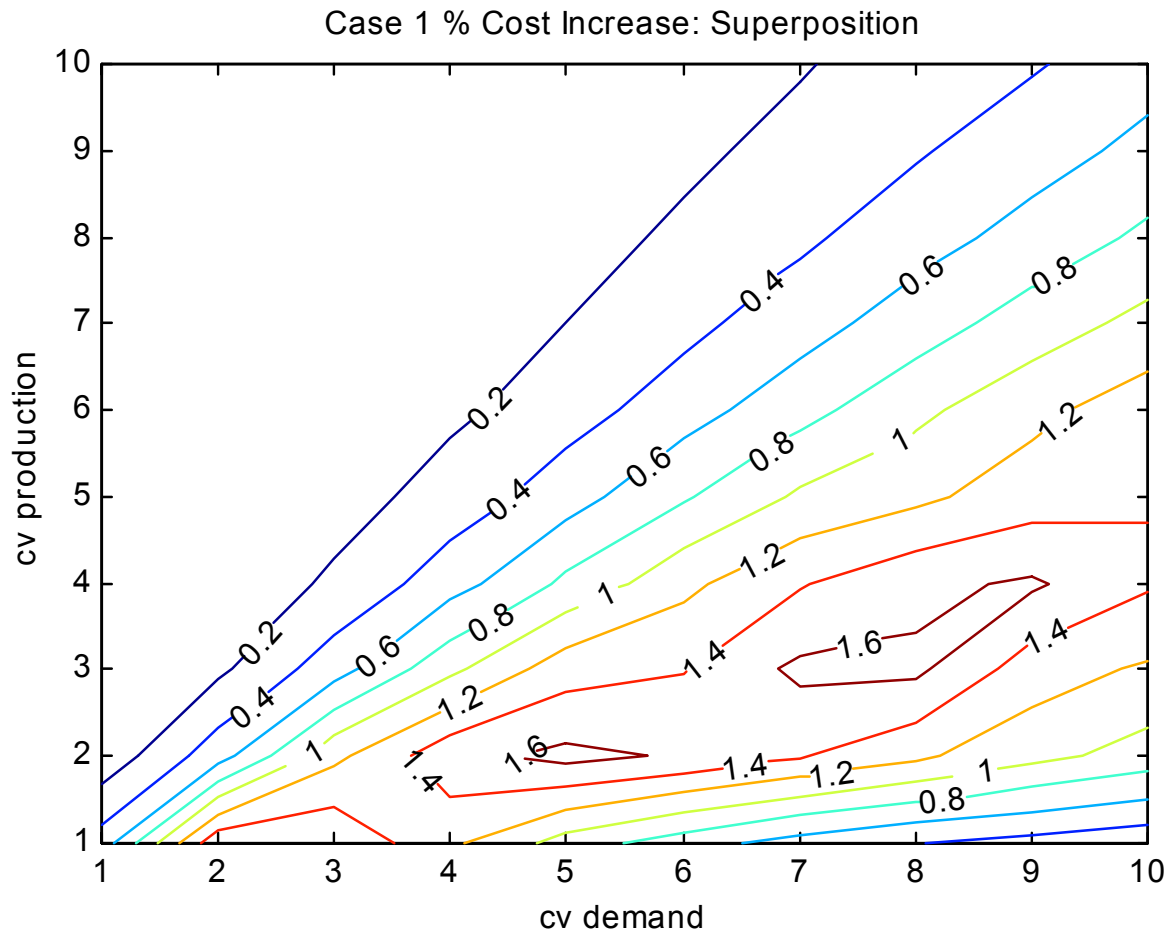

Figure 3. The average cost increase percentage by using the superposition policy instead of the double hedging policy for Case 1: $\mu_{1}>\mu_{2}, \mu_{1}=1.0, E_{1}=0.9, \mu_{2}=0.95, E_{2}=0.8, c^{+}=1, c^{-}=2$.

Case $2 \%$ Cost Increase: Superposition

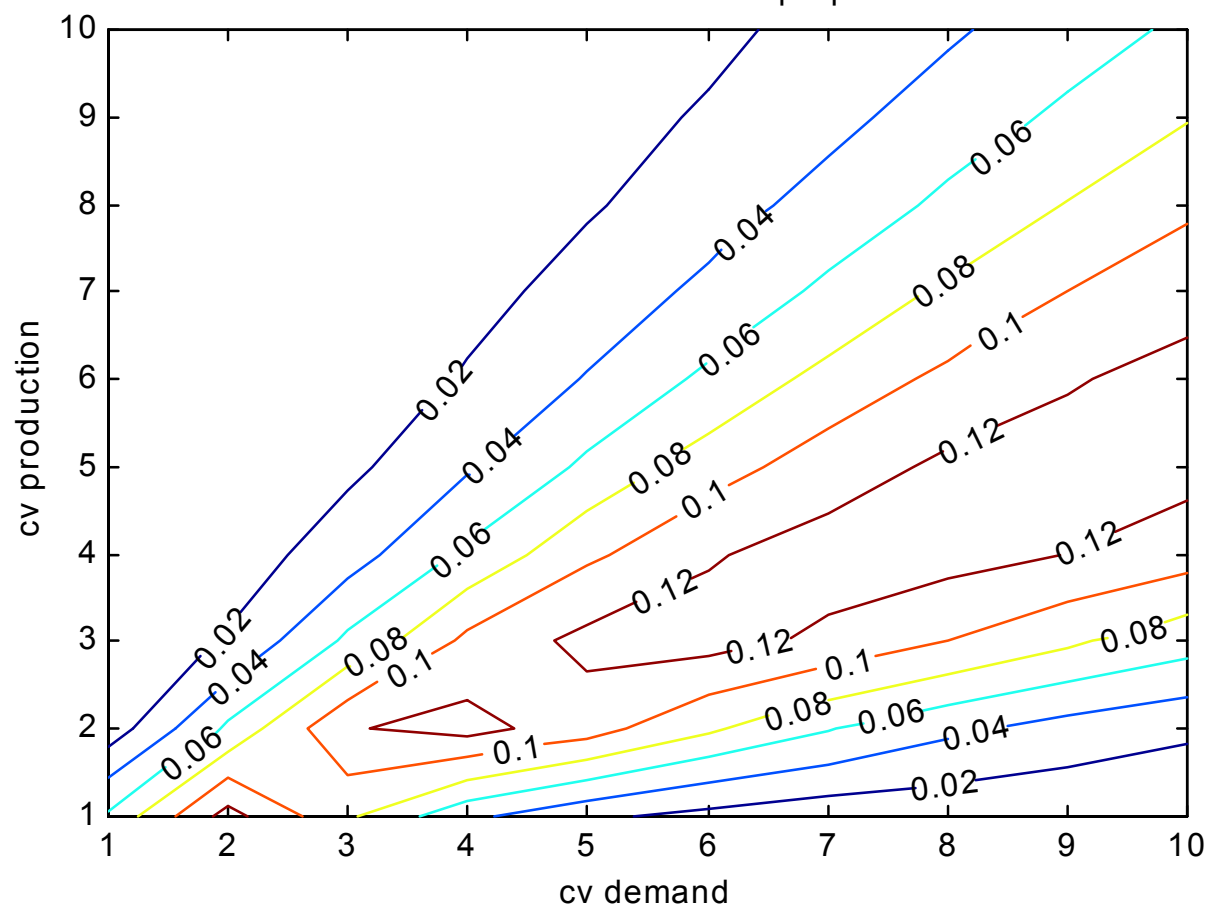

Figure $3 \mathrm{~b}$. The average cost increase percentage by using the superposition policy instead of the double hedging policy for Case $2: \mu_{1}=\mu_{2}, \mu_{1}=1.0, E_{1}=0.9, \mu_{2}=1.0, E_{2}=0.8, c^{+}=1, c^{-}=2$. 


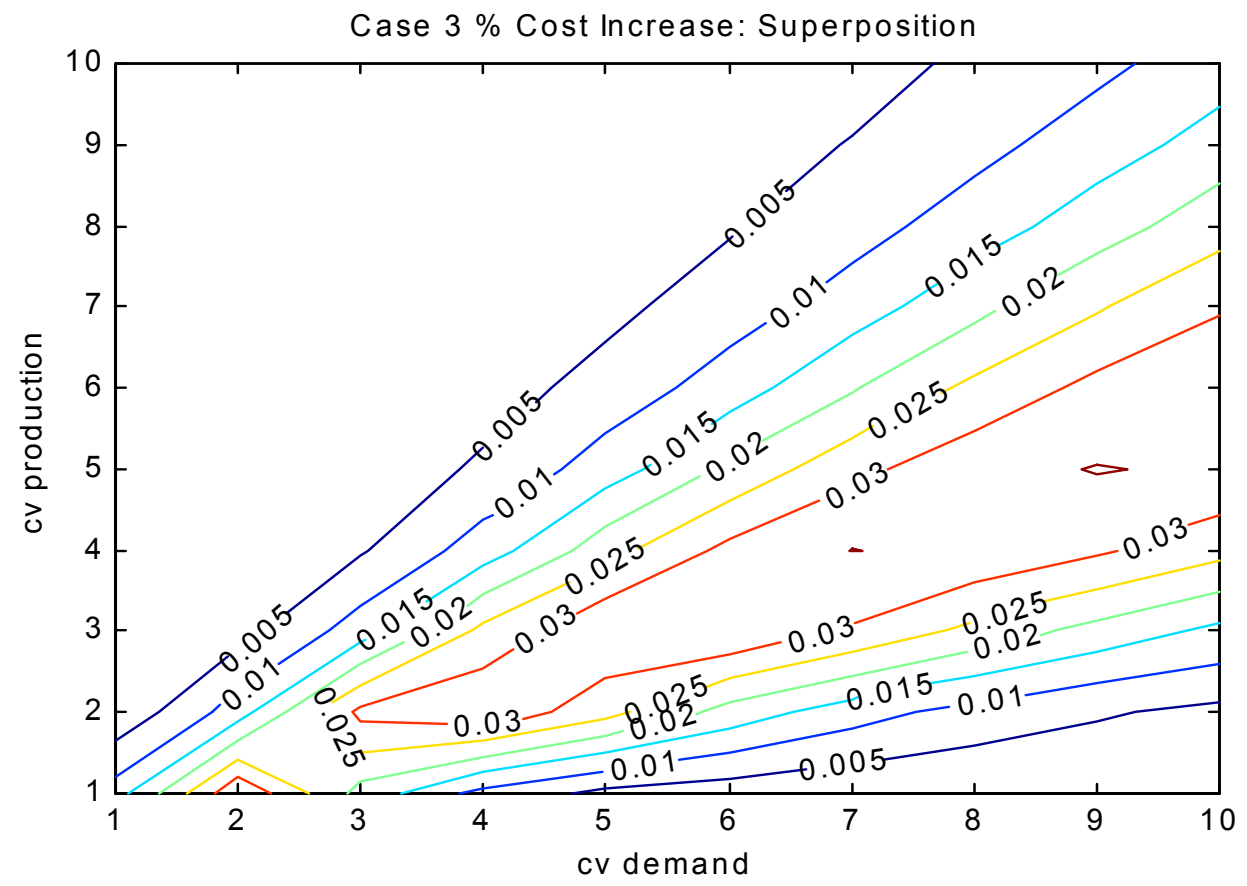

Figure 3c. The average cost increase percentage by using the superposition policy instead of the double hedging policy for Case $3: \mu_{1}<\mu_{2}, \mu_{1}=1.0, E_{1}=0.9, \mu_{2}=1.2, E_{2}=0.8, c^{+}=1, c^{-}=2$

\section{References}

Akella, R. and Kumar, P.R. (1986), "Optimal Control of Production Rate in a Failure Prone Manufacturing System," IEEE Transactions on Automatic Control, Vol. AC-31, No. 2, pp. 116-126.

Algoet, P.H. (1989), "Flow Balance Equations for the Steady-State Distribution of a Flexible Manufacturing System," IEEE Transactions on Automatic Control, Vol. 34, No.8, pp.917-921.

Bielecki, T. and Kumar, P.R. (1988), "Optimality of Zero-Inventory Policies for Unreliable Manufacturing Systems," Operations Research, Vol.36, No. 4, pp. 532-541.

Gershwin, S.B. (1994), Manufacturing Systems Engineering, Englewood Cliffs, New Jersey: PTR Prentice Hall.

Hu, J.Q., P. Vakili, and Y.G. Yu (1994), "Optimality of Hedging Point Policies in the Production Control of Failure-Prone Manufacturing Systems," IEEE Transactions on Automatic Control, Vol. 39, pp. 1875-1880.

Kimemia, J. G. and Gershwin, S. B. (1983), "An Algorithm for the Computer Control of Production in Flexible Manufacturing systems," IIE Transactions, Vol. AC-15, pp. 352-362.

Liberopoulos, G. and M. Caramanis (1994), "Production Control of Manufacturing Systems with Production Rate-Dependent Failure Rates," IEEE Transactions on Automatic Control, Vol. 39, No. 4, pp. 889-895.

Liberopoulos, G. and J.Q. Hu (1995), "On the Ordering of Optimal Hedging Points in a Class of Manufacturing Flow Control Models," IEEE Transactions on Automatic Control, Vol. 40, No. 2, pp. 282-286.

Sharifnia, A. (1988), "Production Control of a Manufacturing system with Multiple Machine States," IEEE Transactions on Automatic Control, Vol. 33, No. 7, pp.620-625.

Tan, B. (1997), "Variance of the Throughput of an N-station Production Line with No Intermediate Buffers and Time Dependent Failures," European Journal of Operational Research, Vol.101, No.3, pp.560-576.

Tan, B. (2000), "Production Control of a Failure Prone Manufacturing system with Variable Demand," Koc University Working Paper Series 00-1, Istanbul, Turkey. 